\title{
Dynamics of poly(ethylene oxide) chains in water
}

\author{
A. Eshuis and P. F. Mijnlieff \\ Rheology Group, Department of Applied Physics, Twente University of Technology, PO \\ Box 217, 7500 AE Enschede, The Netherlands \\ (Received 17 August 1984; revised 7 May 1986)
}

\begin{abstract}
Dynamic moduli of dilute aqueous solutions of four poly(ethylene oxide) samples were measured as a function of frequency at four temperatures. From the measurements on two quite monodisperse samples the value of the Peterlin parameter $\varepsilon$ was determined using a shifting procedure. A definite decrease in $\varepsilon$ was observed when going from $10^{\circ} \mathrm{C}$ to $40^{\circ} \mathrm{C}$. Polydisperse samples were analysed by treating them as consisting of one monodisperse high-molecular mass fraction which does, and a remaining polydisperse lowermolecular mass fraction which does not contribute to the reduced dynamic moduli.
\end{abstract}

(Keywords: dynamic viscoelastic modulus; Mark-Houwink relation; Peterlin parameter; $\theta$-conditions; local association)

\section{INTRODUCTION}

During an investigation into the properties of certain nonionic microemulsions we met an interesting phenomenon. When temperature is increased the microemulsions start to develop a remarkable elasticity above a certain temperature ${ }^{1,2}$. Below that temperature the system properties are those of a dispersion of individual oil droplets in water but the changes taking place above this temperature must be attributed to the formation of long-range structures.

The surfactant stabilizing these emulsions has, as the hydrophilic part, a chain containing 5 to 10 ethylene oxide units. The transformation mentioned above is, we think, in some way or another connected with the influence of temperature on the conformations of the oligo-ethylene oxide chains. In the systems studied the 'transformation temperature' varied between $5^{\circ} \mathrm{C}$ and $25^{\circ} \mathrm{C}$, depending on the surfactant-to-oil ratio.

It is well known that the quality of water as a solvent for poly(ethylene oxide) (PEO) decreases with increasing temperature $^{3}$. Breaking down of hydrogen bonds between the ether groups and water, and destruction of the ice cage around the polymer chains, are believed to be the mechanisms behind this behaviour.

For oligomers of ethylene oxide (molecular masses $M$ of $400,1000,1500$ and 1540) a drastic influence of temperature on their limiting viscosity number $[\eta]$, also known as the 'intrinsic viscosity', in water has been reported $^{4}$. As an example we cite that, for $M=1000$, $[\eta]$ drops from $5.1 \mathrm{ml} / \mathrm{g}$ at $30^{\circ} \mathrm{C}$ to $2.5 \mathrm{ml} / \mathrm{g}$ at $40^{\circ} \mathrm{C}$. Although an interpretation in terms of molecular conformation is not in place at such low $M$-values, this result suggests a considerable shrinking of the oligo-ethylene oxide chains (perhaps by folding on themselves).

It is also known, however, that aqueous PEO solutions have their cloud point, somewhat depending on concentration and molecular mass, close to or even above $100^{\circ} \mathrm{C}^{3}$. Furthermore, limiting viscosity numbers of PEO in water, with $M \gtrsim 4000$, are not exceptionally low as compared with those of other polymers with the same contour length ${ }^{4,5}$. Therefore, when there is some kind of chain folding, it must be localized along the chain, not really affecting the coil-character of the molecule as a whole.

We wondered whether conformational changes with temperature could be recognized in the linear viscoelastic behaviour of dilute PEO solutions in water. Dynamic moduli at a number of frequencies between 4 and $235 \mathrm{kHz}$ were, therefore, determined for some PEO samples at four temperatures.

Values of the exponent $\alpha_{n}$ in the Mark-Houwink relation

$$
[\eta]=K M^{\alpha}{ }^{n}
$$

were obtained using a shifting procedure. Although this procedure could only be applied to two out of the four PEO samples investigated (the other two were quite polydisperse) it left little room for uncertainty about the conclusion with respect to the temperature dependence of $\alpha_{\eta}$. We arrived at a stronger decrease of $\alpha_{\eta}$ with $T$ than did $\mathrm{Amu}^{5}$.

\section{EXPERIMENTAL}

Samples

The molecular masses of the samples as quoted by the supplier were $14000 ; 100000 ; 200000$ (supplier: Janssen Chimica, Beerse, Belgium) and 35000 (supplier:Merck, Darmstadt, West Germany).

We analysed these samples by gel permeation chromatography (g.p.c.) (Waters Model 6000A solvent delivery system; Waters Model R401 differential refractometer; separating columns: TSK: G5000 PW + G4000 PW + G3000 PW in series; solvent: water). This series of columns had been calibrated with a set of PEO samples ('Pharmacia'); for each sample the liquid leaving the last column was sent to the differential refractometer and to a low-angle laser light-scattering instrument (both with 8 microlitre cell contents) to determine the polymer concentration and the polymer molecular mass of the liquid leaving the column. In this 
way the molecular mass-elution volume relationship was established for each sample. A 'broad' calibration curve was constructed from the results for seven samples, with $\bar{M}_{\mathrm{w}}$ values ranging from 3000 to 300000 . This calibration method is the same as that described by Zhi-Duan et al. ${ }^{6}$

Using this calibration curve, the elution curves (refractive index versus elution volume) of the samples were converted into the molecular mass distribution curves shown in Figure 1 . Corresponding average molecular mass data are given in Table 1 .

\section{Determination of dynamic moduli}

Dynamic moduli (see next paragraph) were determined with a nickel tube apparatus. With this instrument, developed recently in our laboratory ${ }^{7}$, dynamic moduli $G^{\prime}$ and $G^{\prime \prime}$ (or, equivalently, dynamic viscosities $\eta^{\prime \prime}$ and $\eta^{\prime}$ ) can be measured at a discrete set of frequencies between 3 and $250 \mathrm{kHz}$. The zero-frequency viscosity $\eta_{0}$ should not exceed $\sim 25 \mathrm{cP}$, and measured $G^{\prime}$ values lower than $\sim 0.05 \times G^{\prime \prime}$ can no longer be considered significant. Measurements were performed on solutions of the samples in water at temperatures $10^{\circ} \mathrm{C}, 20^{\circ} \mathrm{C}, 30^{\circ} \mathrm{C}$ and $40^{\circ} \mathrm{C}$ at a number of concentrations.

Table 1 Molecular mass data of the samples

\begin{tabular}{|c|c|c|c|c|}
\hline $\begin{array}{l}\text { Sample } \\
\text { number }\end{array}$ & $\begin{array}{l}\bar{M} \\
\text { (stated by } \\
\text { supplier) }\end{array}$ & $\bar{M}_{\mathrm{n}}$ & $\bar{M}_{\mathrm{w}}$ & $\frac{\bar{M}_{\mathrm{w}}}{\bar{M}_{\mathrm{n}}}$ \\
\hline 1 & 14000 & 10600 & 12800 & 1.21 \\
\hline 2 & 35000 & 25900 & 29300 & 1.13 \\
\hline 3 & 100000 & 21700 & 118000 & 5.44 \\
\hline 4 & 200000 & 61400 & 187000 & 3.05 \\
\hline
\end{tabular}

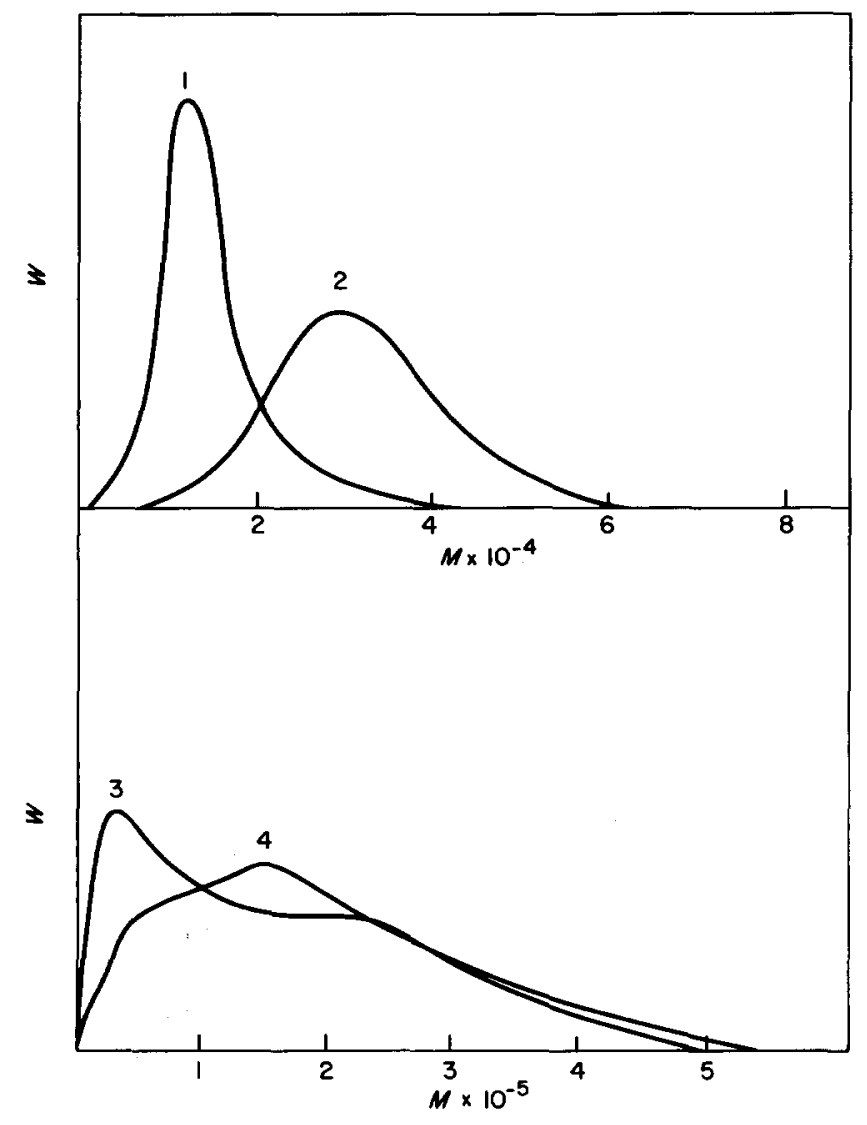

Figure 1 Molecular mass distributions of the PEO samples: (1) $\vec{M}_{\mathrm{w}}=12800 ;(2) \bar{M}_{\mathrm{w}}=29000 ;(3) \bar{M}_{\mathrm{w}}=118000 ;(4) \bar{M}_{\mathrm{w}}=187000$

\section{DYNAMIC MODULI OF POLYMER SOLUTIONS}

When a harmonic shear $\varepsilon(t)$, that is:

$$
\varepsilon(t)=\varepsilon_{0} \sin \omega t=(\operatorname{Re}) \varepsilon_{0} \exp (i \omega t) \equiv(\operatorname{Re}) \hat{\varepsilon}(t)
$$

where $\varepsilon_{0}$ is the amplitude and $\omega$ the angular frequency, is imposed on a linear viscoelastic material, the shear stress $\sigma(t)$ required to maintain the harmonic shear is related to $\varepsilon(t)$ as:

$$
\begin{aligned}
\sigma(t)=(\operatorname{Re})\left[G^{*}(i \omega) \varepsilon(t)\right]=(\operatorname{Re})\left[G^{*}(i \omega)\right. & \left.\frac{1}{i \omega} \hat{\dot{\varepsilon}}(t)\right] \\
& =(\operatorname{Re})\left[\eta^{*}(i \omega) \hat{\dot{\varepsilon}}(t)\right]
\end{aligned}
$$

where $i=(-1)^{1 / 2}$, and $G^{*}(i \omega)$ is the complex shear modulus, in which the dot denotes a time derivative and where $\eta^{*}(i \omega)$ is the complex shear viscosity. $G^{*}(i \omega)$ and $\eta^{*}(i \omega)$ are generally broken down to:

$$
G^{*}(i \omega)=G^{\prime}+i G^{\prime \prime} ; \eta^{*}(i \omega)=\eta^{\prime}-i \eta^{\prime \prime}
$$

From equations (2)-(4) it follows that the dynamic moduli $G^{\prime}$ and $G^{\prime \prime}$ are related to the dynamic viscosities $\eta^{\prime}$ and $\eta^{\prime \prime}$ by:

$$
G^{\prime}=\omega \eta^{\prime \prime} ; G^{\prime \prime}=\omega \eta^{\prime}
$$

From the general theory of linear dissipative liquid systems it follows that $G^{\prime}$ and $G^{\prime \prime}$ can always be written as:

$$
G^{\prime}=\sum_{p=1}^{z} G_{p} \frac{\omega^{2} \tau_{p}^{2}}{1+\omega^{2} \tau_{p}^{2}} ; G^{\prime \prime}=\omega \eta_{\infty}+\sum_{p=1}^{z} G_{p} \frac{\omega \tau_{p}}{1+\omega^{2} \tau_{p}^{2}}
$$

in which $\eta_{\infty}$ is a constant and $G_{p}$ and $\tau_{p}$ denote the strength and the relaxation time of the $p$-th relaxation mechanisms, of which mechanisms there are $z$ in total.

The analysis of our results will be based on a few general conclusions of the various molecular theories on the linear viscoelasticity of polymer solutions at the limit of infinite dilution ${ }^{8,9}$. One of these conclusions is:

$$
G_{p}=v R T=\frac{c}{M} R T \quad(\text { all } p)
$$

where $R$ is the gas constant and $T$ the absolute temperature, and where $v$ and $c$ are the number of moles and the number of mass units of the polymer per unit volume of the solution, respectively.

Another conclusion is:

$$
\eta_{\infty}=\eta_{s}
$$

where $\eta_{s}$ is the viscosity of the solvent (in this case water, which is considered a Newtonian liquid at all frequencies applied). These conclusions hold for 'sufficiently' flexible polymers, a condition which we will assume to be satisfied. According to existing theories, for nonbranched polymers all $\tau_{p}$ are different, and their values rapidly decrease with increasing $p$. The number of relaxation mechanisms, $N$, is proportional to the polymer molecular mass. For most practical purposes $N$ may be considered to be large (say $N>50$ ) at low molecular masses (say $M>5000$ ). From now on we will replace $N$ by $\infty$ throughout. 
Combining equations (6)-(8) one finds:

$$
G^{\prime}=\frac{R T c}{M} \sum_{p=1}^{\infty} \frac{\omega^{2} \tau_{p}^{2}}{1+\omega^{2} \tau_{p}^{2}} ; G^{\prime \prime}=\omega \eta_{s}+\frac{R T c}{M} \sum_{p=1}^{\infty} \frac{\omega \tau_{p}}{1+\omega^{2} \tau_{p}^{2}}
$$

for a monodisperse polymer at infinite dilution.

For further analysis, values of the so-called intrinsic quantities are required. These are denoted by $\left[\eta^{\prime}\right]$, etc., and defined as:

$$
\begin{gathered}
{\left[\eta^{\prime}\right] \equiv \lim _{c \rightarrow 0}\left\{\frac{\eta^{\prime}-\eta_{s}}{\eta_{s} c}\right\}=\lim _{c \rightarrow 0}\left\{\frac{1}{\omega \eta_{s}} \cdot \frac{G^{\prime \prime}-\omega \eta_{s}}{c}\right\} \equiv \frac{1}{\omega \eta_{s}}\left[G^{\prime \prime}\right]} \\
{\left[\eta^{\prime \prime}\right] \equiv \lim _{c \rightarrow 0}\left\{\frac{\eta^{\prime \prime}}{\eta_{s} c}\right\}=\lim _{c \rightarrow 0}\left\{\frac{1}{\omega \eta_{s}} \cdot \frac{G^{\prime}}{c}\right\} \equiv \frac{1}{\omega \eta_{s}}\left[G^{\prime}\right]}
\end{gathered}
$$

The quantities $\left\{\frac{\eta^{\prime}-\eta_{s}}{\eta_{s} c}\right\}$ and $\left\{\frac{\eta^{\prime \prime}}{\eta_{s} c}\right\}$ will be called the dynamic viscosity numbers. $[\eta]$ :

For $\omega \rightarrow 0,\left[\eta^{\prime}\right]$ reduces to the limiting viscosity number

$$
\left[\eta^{\prime}\right]_{\omega \rightarrow 0}=[\eta]
$$

Following Ferry ${ }^{10}$, we now introduce reduced moduli, defined as:

$$
\begin{aligned}
{\left[G^{\prime}\right]_{\mathrm{R}} } & \equiv \frac{M}{R T}\left[G^{\prime}\right]=\omega \eta_{s} \frac{M}{R T}\left[\eta^{\prime \prime}\right] ; \\
{\left[G^{\prime \prime}\right]_{\mathrm{R}} } & \equiv \frac{M}{R T}\left[G^{\prime \prime}\right]=\omega \eta_{s} \frac{M}{R T}\left[\eta^{\prime}\right]
\end{aligned}
$$

Molecular theories may result in predictions about the dependence of these dimensionless quantities on $\omega$.

\section{RESULTS FOR $G^{\prime}$ AND $G^{\prime \prime}$}

Solutions of polymers with, say e.g. $M=10^{5}$, in ordinary low-viscous solvents have a longest relaxation time not exceeding a few tenths of a millisecond. This follows from various molecular theories (differing from each other in the precise numerical predictions about the $\tau_{p}$ 's only), and also from experiments on polymer solutions themselves. The latter experiments were mostly performed in highly viscous solvents in order to 'scale up' relaxation times, thus lowering the frequency region where the storage modulus $G^{\prime}$ initially becomes measurable. Being interested in the particular properties of water as a solvent for PEO we could not apply such a procedure. Fortunately, we did have available the nickel-tube apparatus, designed for measurements in the $\mathrm{kHz}$ region ${ }^{7}$. All dynamic viscosities were determined with this instrument, after which they were converted into dynamic viscosity numbers.

In Figure 2 examples are shown of the extrapolation of these quantities towards zero concentration in order to obtain the values of the intrinsic quantities defined in equations (10). In these extrapolations linearity between $\left(\eta^{\prime}-\eta_{s}\right) /\left(\eta_{s} c\right)$ and $c$, and between $\eta^{\prime \prime}\left(\eta_{s} c\right)$ and $c$, is assumed. More sophisticated extrapolations have been proposed ${ }^{11}$ where the above plots do not yield straight lines. Owing to the low concentrations at which we could make measurements, however, our results do not highlight the need for the applications of these extrapolations. The intrinsic dynamic viscosities obtained were converted into reduced moduli defined in equations (12); for $M$ the $\bar{M}_{\mathrm{w}}$ values (Table 1$)$ of the samples were used.

In Figure 3 some examples of double logarithmic plots of $\left[G^{\prime}\right]_{\mathrm{R}}$ and $\left[G^{\prime \prime}\right]_{\mathrm{R}}$ versus frequency are given. These results will be analysed in the following section.

\section{ANALYSIS OF RESULTS}

With the aid of equations (9) and (10) the reduced moduli $\left[G^{\prime}\right]_{\mathrm{R}}$ and $\left[G^{\prime \prime}\right]_{\mathrm{R}}$ defined in equations $(12)$, can be written as:

$$
\begin{gathered}
{\left[G^{\prime}\right]_{\mathrm{R}}=\sum_{p=1}^{\infty} \frac{\omega^{2} \tau_{p}^{2}}{1+\omega^{2} \tau_{p}^{2}}=\sum_{p=1}^{\infty} \frac{\left(\omega \tau_{1}\right)^{2}\left(\tau_{p} / \tau_{1}\right)^{2}}{1+\left(\omega \tau_{1}\right)^{2}\left(\tau_{p} / \tau_{1}\right)^{2}}} \\
{\left[G^{\prime \prime}\right]_{\mathrm{R}}=\sum_{p=1}^{\infty} \frac{\omega \tau_{p}}{1+\omega^{2} \tau_{p}^{2}}=\sum_{p=1}^{\infty} \frac{\left(\omega \tau_{1}\right)\left(\tau_{p} / \tau_{1}\right)}{1+\left(\omega \tau_{1}\right)^{2}\left(\tau_{p} / \tau_{1}\right)^{2}}}
\end{gathered}
$$

We now assume, as is usual ${ }^{10}$, that the quantities $\left(\tau_{p} / \tau_{1}\right)$ are independent of molecular mass. This implies that the influence of polymer molecular mass on the reduced moduli given by equations (13) is achieved through its influence on the longest relaxation time, $\tau_{1}$, only. If we now consider samples with molecular masses $M_{a}$ and $M_{b}$, the longest relaxation times, at infinite dilution, will be $\tau_{1, a}$ and $\tau_{1, b}$. From the above assumption, applied to equations (13), it then follows that the frequency, $\omega_{b}$, at which the reduced moduli of the solution of sample ' $b$ ' equal those at frequency $\omega_{a}$ of the solution of sample ' $a$ ' is given by:

$$
\omega_{b}=\frac{\tau_{1, a}}{\tau_{1, b}} \omega_{a}
$$

Plots of $\left[G^{\prime}\right]_{\mathrm{R}}$ and $\left[G^{\prime \prime}\right]_{\mathrm{R}}$ (or of $\log \left[G^{\prime}\right]_{\mathrm{R}}$ and $\log \left[G^{\prime \prime}\right]_{\mathrm{R}}$ ) versus $\log \omega$ for sample ' $b$ ' can therefore be brought to coincide with those for sample ' $a$ ' by being shifted over a distance, $\Delta^{b, a} \log \omega$, given by:

$$
\Delta^{b, a} \log \omega=\log \frac{\tau_{1, b}}{\tau_{1, a}}
$$

along the $\log \omega$ axis. This is a well-known procedure to bring curves of the dynamic moduli for samples with different molecular masses on two single master curves. If the procedure appears to apply, the assumptions underlying it are, at least, not contradicted, and the master curves may then be analysed, as discussed by Ferry ${ }^{10}$, for checking certain predictions about the set of quantities $\left(\tau_{p} / \tau_{1}\right)$. The measured shifts themselves, however, also contain some useful information. Combining equations (10a), (11), (12b) and (13b) one finds:

$$
[\eta]=\frac{R T}{\eta_{s} M} \sum_{p=1}^{\infty} \tau_{p}=\frac{R T \tau_{1}}{\eta_{s} M} \sum_{p=1}^{\infty} \frac{\tau_{p}}{\tau_{1}}
$$

For $[\eta]$ we use the Mark-Houwink relation (equation (1)), in which we replace $\alpha_{\eta}$ by $1 / 2+(3 / 2) \varepsilon$, see e.g. Elias ${ }^{12}$; for random-flight statistics, or at $\theta$-conditions, the 

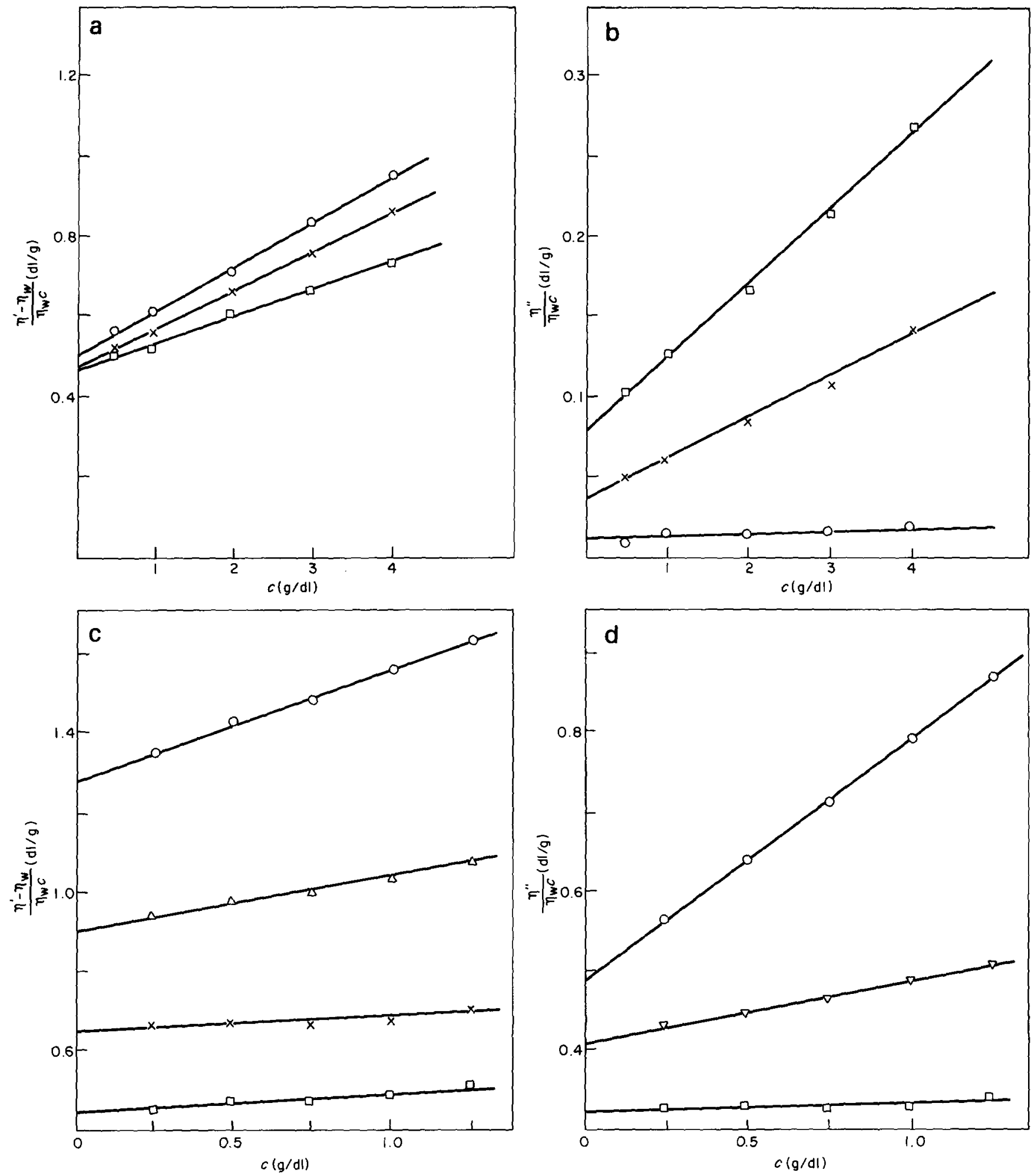

Figure 2 Extrapolation of the dynamic viscosity numbers towards zero concentration. The two lower figures (c and d) are for $\bar{M}_{\mathrm{w}}=187000$ at $T=10^{\circ} \mathrm{C}$, the two upper ones $(\mathrm{a}$ and $\mathrm{b})$ for $\bar{M}_{\mathrm{w}}=29300$ at $T=40^{\circ} \mathrm{C}$. Frequencies: $(O), \leftrightarrow 11200 \mathrm{~Hz} ;(\triangle), \leftrightarrow 33680 ;(\nabla)$ and $(\times) \leftrightarrow 78620 \mathrm{~Hz}$; $(+) \leftrightarrow 101100 \mathrm{~Hz} ;(\square) \leftrightarrow 235980 \mathrm{~Hz}$

Peterlin parameter $\varepsilon$ equals zero. Combining equations (1) and (15) we then find:

$$
\tau_{1}=\frac{\eta_{s}[\eta] M}{R T \sum_{p=1}^{\infty} \frac{\tau_{p}}{\tau_{1}}}=\frac{\eta_{s} K M^{3 / 2+(3 / 2) \varepsilon}}{R T \sum_{p=1}^{\infty} \frac{\tau_{p}}{\tau_{1}}}
$$

For one and the same polymer-solvent pair the quantities $\eta_{s}, K, \varepsilon$ and $\sum_{p=1}^{\infty}\left(\tau_{p} / \tau_{1}\right)$ are dependent only on temperature.

Substituting equation (16), for $M=M_{a}$ and for $M=M_{b}$, into equation (14) we find:

$$
\Delta^{b, a} \log \omega=\left[\frac{3}{2}+\frac{3}{2} \varepsilon\right] \log \frac{M_{b}}{M_{a}}
$$

Therefore, from measured frequency shifts, with $M_{a}$ and $M_{b}$ known, $\varepsilon$-values can be derived. 

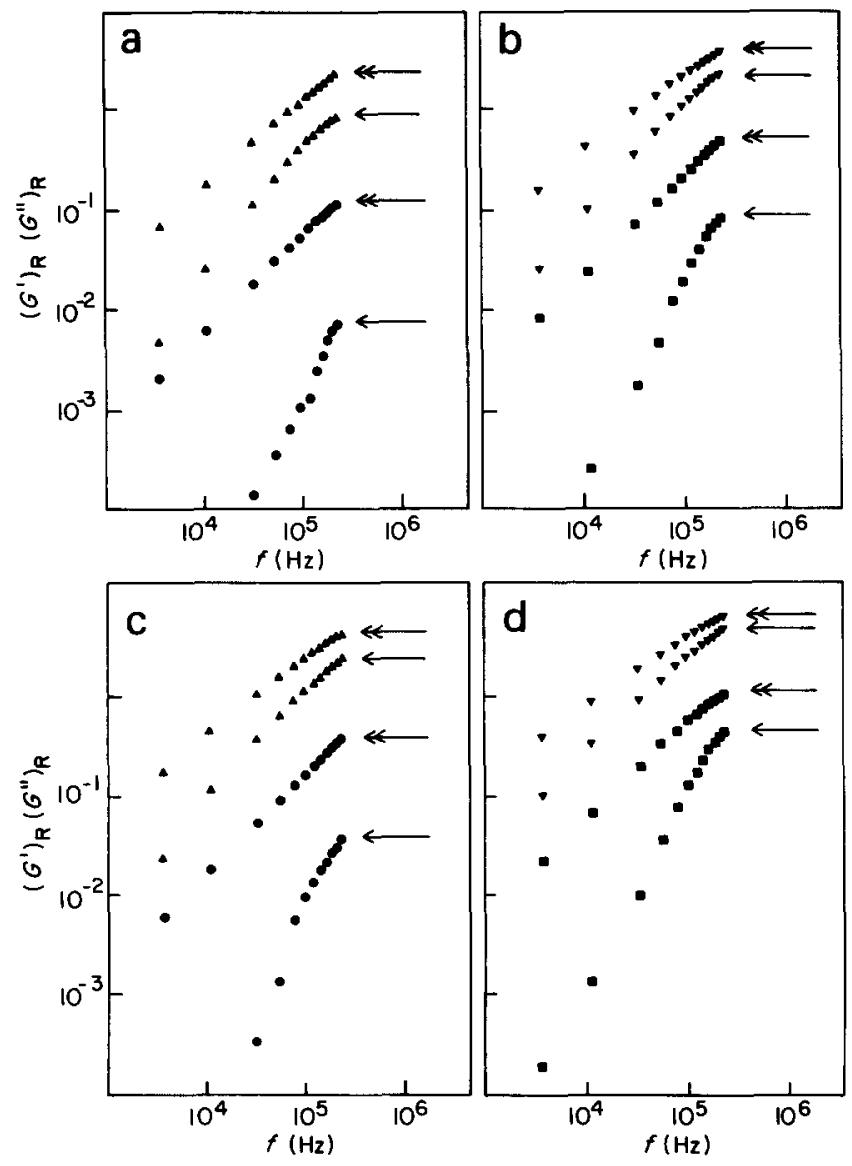

Figure 3 Reduced moduli of the four samples at $40^{\circ} \mathrm{C}$ (a and b) and $10^{\circ} \mathrm{C}$ (c and $\mathrm{d}$ ). The arrows $\leftarrow$ and $\leftarrow$ indicate $\left[G^{\prime}\right]_{\mathrm{R}}$ and $\left[G^{\prime \prime}\right]_{\mathrm{R}}$, respectively. Molecular masses: (O), $\leftrightarrow \bar{M}_{\mathrm{w}}=12800$; (G) $\leftrightarrow \bar{M}_{\mathrm{w}}=29300 ;(\mathbf{\Lambda}), \leftrightarrow \bar{M}_{\mathrm{w}}=118000 ;(\nabla), \leftrightarrow \bar{M}_{\mathrm{w}}=187000$

We will now investigate the reduced-moduli plots, some of which are shown in Figure 3.

At each one of the four temperatures at which measurements were performed, it appears that only the plots for the two lower-molecular-mass samples (1 and 2) can be brought to coincidence by means of a horizontal shift alone. The master plots obtained, at $10^{\circ} \mathrm{C}$, are shown in Figure 4, which will be explained later on.

The shifts at all four temperatures are given in Table 2, together with the $\varepsilon$-values derived from the shifts by means of equation (17). From these results, with the estimated error bounds indicated, we observe that $\varepsilon$ definitely decreases with increasing temperature in the range covered. It is true that this conclusion is based on only one horizontal shift at each temperature, but it is, however, the shift of a whole collection of points and not just that of a single point.

The plots of the two higher molecular mass samples do not allow for such a simple analysis. It appears that these plots can only be brought to coincide with those for the two lower molecular mass samples by adding to a horizontal shift in the positive $\log \omega$ direction a vertical shift in the $\log \left[G^{\prime}\right]_{\mathbf{R}}$ or $\log \left[G^{\prime \prime}\right]_{\mathbf{R}}$ direction. We ascribe this effect to the high degree of polydispersity (see Figure l) of the two high molecular mass samples.

If the set of quantities $\tau_{p} / \tau_{1}$ were known as a function of temperature then, on using the known values of $\varepsilon$ and the measured molecular mass distribution, the shape $(K$ is not needed for this) of the curves of $\log \left[G^{\prime}\right]_{\mathrm{R}}$ and $\log \left[G^{\prime \prime}\right]_{\mathrm{R}}$ as a function of $\log \omega$ could be calculated. Calculations of

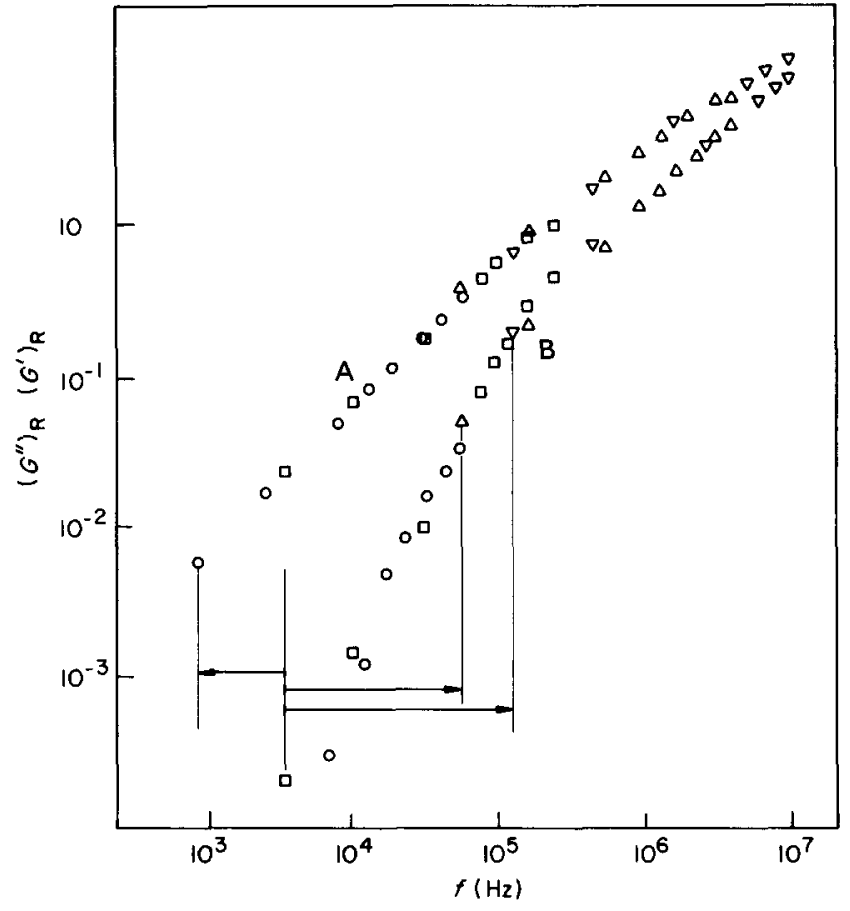

Figure 4 Results of shifting procedure applied to the dynamic moduli plots at $10^{\circ} \mathrm{C} . \mathrm{A},\left[G^{\prime \prime}\right]_{\mathrm{R}} ; \mathrm{B},\left[G^{\prime}\right]_{\mathrm{R}} \cdot(\mathrm{O})$, results for sample 1 (shifted horizontally); $\square$ ), results for sample 2 (not shifted); $\triangle$ ), results for sample 3 (shifted horizontally and vertically); $(\nabla)$, results for sample 4 (shifted horizontally and vertically)

Table 2 Peterlin parameter $\varepsilon$ at different temperatures as derived from the horizontal shifts $\Delta^{1,2} \log \omega$

\begin{tabular}{lll}
\hline $\begin{array}{l}\text { Temperature } \\
\left({ }^{\circ} \mathrm{C}\right)\end{array}$ & $\begin{array}{l}\Delta^{1,2} \log \omega \\
\pm 0.01\end{array}$ & $\begin{array}{l}\varepsilon \\
\pm 0.02\end{array}$ \\
\hline 10 & 0.63 & 0.17 \\
20 & 0.61 & 0.13 \\
30 & 0.58 & 0.08 \\
40 & 0.56 & 0.04 \\
\hline
\end{tabular}

this type have already been carried out ${ }^{10}$. We did not wish, however, to perform such a calculation as long as it would contain an assumption* about the $\left(\tau_{p} / \tau_{1}\right)$ 's, and prefer, for the time being, to analyse the observed effects in the following simplistic way.

From equation (16) it can be seen that the longest relaxation time, whatever the precise value of $\varepsilon$, strongly increases with increasing $M$. Consequently, in a polydisperse sample, at concentrations sufficiently low for the contributions of the various fractions to $G^{\prime}$ and $G^{\prime \prime}$ to be additive, the influence of the higher molecular mass fractions strongly dominate. We have, therefore, decided to treat $G^{\prime}$ and $G^{\prime \prime}$ as the contribution of just one monodisperse fraction, with molecular mass $M_{\mathrm{H}}$ and weight fraction $w_{\mathrm{H}}$, of the polydisperse sample. According to the first of equations (9), $G^{\prime}$ is then given by:

$$
G^{\prime}=\frac{c_{\mathrm{H}}}{M_{\mathrm{H}}} R T \sum_{p=1}^{\infty} \frac{\left(\omega \tau_{p, \mathrm{H}}\right)^{2}}{1+\left(\omega \tau_{p, \mathrm{H}}\right)^{2}}
$$

* It has been proposed recently (P. F. Mijnlieff in 'Advances in R heology [Elsevier Science Publishers, 1984] Vol. I, pp. 459-465) that $\tau_{p} / \tau_{1}=p^{-(3 / 2)(1+\varepsilon)}$. Careful checks on this prediction are now being carried out. When the prediction is confirmed, calculation of dynamic moduli will be performed for the polydisperse samples. 
where $c_{\mathrm{H}}$ is the concentration of the fraction with $M=M_{\mathrm{H}}$. Note that

$$
c_{\mathrm{H}}=w_{\mathrm{H}} c
$$

An expression analogous to equation (18) holds for $G^{\prime \prime}-\omega \eta_{s}$.

The curves in Figure 3 for the reduced moduli were obtained using the weighed-in concentrations $c$ and the weight-averaged molecular masses $\bar{M}_{\mathrm{w}}$ of these samples. In view of equations (18) and (19) this means that for polydisperse samples the reduced moduli as obtained have the following meaning:

$\left[G^{\prime}\right]_{\mathrm{R}}=w_{\mathrm{H}} \frac{\bar{M}_{\mathrm{w}}}{M_{\mathrm{H}}} \sum_{p=1}^{\infty} \frac{\left(\omega \tau_{p, \mathrm{H}}\right)^{2}}{1+\left(\omega \tau_{p, \mathrm{H}}\right)^{2}}=w_{\mathrm{H}} \frac{\bar{M}_{\mathrm{H}}}{M_{\mathrm{H}}} \sum_{p=1}^{\infty} \frac{\left(\omega \tau_{1, \mathrm{H}}\right)^{2}\left(\frac{\tau_{p}}{\tau_{1}}\right)^{2}}{1+\left(\omega \tau_{1, \mathrm{H}}\right)^{2}\left(\frac{\tau_{p}}{\tau_{1}}\right)^{2}}$

$$
\left[G^{\prime \prime}\right]_{\mathrm{R}}=w_{\mathrm{H}} \frac{\bar{M}_{\mathrm{w}}}{M_{\mathrm{H}}} \sum_{p=1}^{\infty} \frac{\left(\omega \tau_{1, \mathrm{H}}\right)\left(\frac{\tau_{p}}{\tau_{1}}\right)}{1+\left(\omega \tau_{1, \mathrm{H}}\right)^{2}\left(\frac{\tau_{p}}{\tau_{1}}\right)^{2}}
$$

The logarithm of the storage modulus of a polydisperse sample is thus given by:

$$
\log \left[G^{\prime}\right]_{\mathrm{R}}=\log w_{\mathrm{H}} \frac{\bar{M}_{\mathrm{w}}}{M_{\mathrm{H}}}+\log \sum_{p=1}^{\infty} \frac{\left(\omega \tau_{1, \mathrm{H}}\right)^{2}\left(\frac{\tau_{p}}{\tau_{1}}\right)^{2}}{1+\left(\omega \tau_{1, \mathrm{H}}\right)^{2}\left(\frac{\tau_{p}}{\tau_{1}}\right)^{2}}
$$

An analogous expression holds for $\log \left[G^{\prime \prime}\right]_{R}$.

From equation (13a) it follows that the corresponding quantity for a monodisperse polymer ' $a$ ' (with $M_{a}$ and $\left.\tau_{1, a}\right)$ is:

$$
\log \left[G^{\prime}\right]_{\mathrm{R}, a}=\log \sum_{p=1}^{\infty} \frac{\left(\omega \tau_{1, a}\right)^{2}\left(\frac{\tau_{p}}{\tau_{1}}\right)^{2}}{1+\left(\omega \tau_{1, a}\right)^{2}\left(\frac{\tau_{p}}{\tau_{1}}\right)^{2}}
$$

From equations (17), (20) and (21) we see that the plot of $\log \left[G^{\prime}\right]_{\mathrm{R}}$ of a polydisperse sample can be brought to coincidence with that of $\log \left[G^{\prime}\right]_{\mathrm{G}, a}$ of a monodisperse sample by applying to it a horizontal shift along the $\log \omega$ axis given by:

$$
\text { 'horizontal shift' }=\left(\frac{3}{2}+\frac{3}{2} \varepsilon\right) \log \frac{M_{\mathrm{H}}}{M_{a}}
$$

plus a vertical shift along the $\log \left[G^{\prime}\right]_{\mathrm{R}}$ axis given by:

$$
\text { 'vertical shift' }=-\log w_{\mathrm{H}} \frac{\bar{M}_{\mathrm{w}}}{M_{\mathrm{H}}}
$$

Figure 4 shows the result of the shifting procedure applied to the plots of $\log \left[G^{\prime}\right]_{R}$ and $\log \left[G^{\prime \prime}\right]_{R}$, at $10^{\circ} \mathrm{C}$, of the four samples. Comparable figures are obtained at the other temperatures.

The $\log \left[G^{\prime}\right]_{\mathrm{R}}$ and $\log \left[G^{\prime \prime}\right]$ plots of sample 2 , taken as the monodisperse reference material ' $a$ ' with $M_{a}=29300$,
Table 3 Values of $M_{H}$ of the two polydisperse samples as derived from the horizontal shifts at four temperatures (Tables $a$ and $b$ ) and the weight fraction $w_{H}$ of the 'heavy' component of these samples as derived from the temperature independent vertical shifts (Table $c$ )

\begin{tabular}{llll}
\hline $\begin{array}{l}\text { a } \\
T\left({ }^{\circ} \mathrm{C}\right)\end{array}$ & $\begin{array}{l}\text { Horizontal } \\
\text { shift }( \pm 0.02)\end{array}$ & $M_{\mathrm{H}}$ & $\begin{array}{l}\text { Overall results } \\
\text { for } \boldsymbol{M}_{\mathrm{H}}\end{array}$ \\
\hline 10 & 1.20 & $134000-149000$ & (sample 3) \\
20 & 1.16 & $134000-150000$ & $150000 \pm$ \\
30 & 1.14 & $140000-158000$ & 20000 \\
40 & 1.15 & $151000-170000$ & \\
\hline
\end{tabular}

\begin{tabular}{llll}
\hline $\begin{array}{l}\text { b } \\
T\left({ }^{\circ} \mathrm{C}\right)\end{array}$ & $\begin{array}{l}\text { Horizontal } \\
\text { shift }( \pm 0.02)\end{array}$ & $M_{\mathrm{H}}$ & $\begin{array}{l}\text { Overall results } \\
\text { for } M_{\mathrm{H}}\end{array}$ \\
\hline 10 & 1.55 & $210000-238000$ & (sample 4) \\
20 & 1.53 & $220000-250000$ & $250000 \pm$ \\
30 & 1.51 & $234000-267000$ & 40000 \\
40 & 1.49 & $246000-284000$ & \\
\hline
\end{tabular}

\begin{tabular}{llll}
\hline c & Vertical & $w_{\mathrm{H}}$ & \\
$\bar{M}_{\mathrm{w}}$ & shift $( \pm 0.01)$ & $M_{\mathrm{H}}$ & $w_{\mathrm{H}}$ \\
\hline 118000 & 0.31 & $(4.17 \pm 0.10) \times 10^{-6}$ & $0.63 \pm 0.10$ \\
187000 & 0.31 & $(2.63 \pm 0.06) \times 10^{-6}$ & $0.66 \pm 0.12$ \\
\hline
\end{tabular}

have not been shifted. Those of the other samples have been shifted horizontally the distance, and in the direction, indicated by the arrows. To the plots of samples 3 and 4 an additional vertical shift (which happened to be the same for these samples) has been applied.

Horizontal and vertical shifts, and conclusions derived therefrom, have been collected in Tables 2 and 3 . Those derived from the shifts for sample 1 were briefly discussed already (Table 2). The horizontal shifts for sample 3 (Table $3 a$ ) and sample 4 (Table $3 b$ ) were interpreted using equation (22), while using, at each temperature, the $\varepsilon$ value given in Table 2. This resulted in the $M_{\mathrm{H}}$ values given in Tables $3 a$ and $b$. The vertical shifts for samples 3 (these shifts appear not to be dependent on temperature), given in Table 3c, were analysed using equation (23). This led to the results, also given for $w_{\mathrm{H}} / M_{\mathrm{H}}$ and those for $w_{\mathrm{H}}$; the latter obtained while using the overall results for $M_{\mathrm{H}}$ given in Tables $3 a$ and $b$.

\section{DISCUSSION}

In this paper we wished to investigate the influence of temperature on the conformation of poly(ethylene oxide) in water. Results were obtained for the temperature dependence of the Peterlin parameter $\varepsilon$ (or, alternatively, the Mark-Houwink exponent $\alpha_{\eta}$ with $\alpha_{\eta}=1 / 2+(3 / 2) \varepsilon$. Usually this type of information is derived from measurements of limiting viscosity numbers or from light scattering. We, however, derived data from measurements of the dynamic moduli at a number of frequencies between 4 and $250 \mathrm{kHz}$.

We wish to again stipulate that the only assumption underlying the procedure followed is the same one as applied in the usual reduced moduli analysis as outlined by Ferry ${ }^{10}$. The assumption is that, for one and the same polymer-solvent pair at one and the same temperature, the set of quantities $\tau_{p} / \tau_{1}$ is independent of polymer molecular mass. It then follows that the shape of the 
curves of the reduced moduli (or their logarithms) versus the logarithm of frequency is the same for all monodisperse samples.

The shifts to be applied to bring the curves for the different samples on to one master curve are directly related (see equation (17)) to the value of the Peterlin parameter $\varepsilon$. We derived $\varepsilon$ values from measurements on two samples only. The uncertainties in the observed shifts were small, however, thus leaving an uncertainty in $\varepsilon$ which does not affect the conclusion reached concerning the general trend in the influence of temperature on $\varepsilon$ (see Table 2).

The behaviour of the curves for the two polydisperse samples was more complicated and we did not derive information about $\varepsilon$ from it. Instead, we analysed these curves as being those of samples containing two or more components, one of which does and the remaining of which do not display viscoelasticity in the frequency range covered. In spite of this analysis being based on an oversimplification, its results (see Table 3) are quite consistent: the vertical shifts do not depend systematically on temperature, whereas the plots themselves do.

We will now briefly discuss the $\varepsilon$-results themselves. They differ from those reported by $\mathrm{Amu}^{5}$ who arrives at a considerably smaller decrease of $\varepsilon$ with $T$ than these authors. We have no explanation for this difference but we do note that Amu's results are based on plots of $\log [\eta]$ versus the logarithm of the number-averaged molecular mass of the samples. For a polydisperse sample, however, the molecular mass $\left(\bar{M}_{\alpha_{n}}\right)$ for which the Mark-Houwink relation holds, is:

$$
\bar{M}_{\alpha_{n}}=\left\{\sum_{i} M_{i}^{\alpha_{n}} w_{i}\right\}^{1 / \alpha_{n}}
$$

For $\alpha_{n} \geqslant 1 / 2$ this molecular mass is closer to the weightaveraged molecular mass than to the number-averaged molecular mass.

As for the decrease of $\varepsilon$ with $T$, indicating polymer coil shrinking, we note that this may be accompanied or caused by local associations within the coil itself.
Evidence for such associations was put forward in 1971 when it was shown that, at one and the same polymer concentration, poly( $\alpha$-methylstyrene) was much more permeable for a poor solvent (cyclohexane) at the $\theta$ temperature than it was for a good solvent (toluene) ${ }^{13}$.

In the specific case of PEO in water, helix formation ${ }^{5}$ or some tendency for local crystallization might promote local associations. For not too low molecular masses this will not affect the coil character of the dissolved polymer molecules, and their intrinsic viscosity will obey a MarkHouwink relation with an $\alpha_{\eta}$ of about 0.5 . For very short chains, however, like those studied by Sandhu and $\mathrm{Jit}^{4}$, the formation of one or two loops as a reaction upon a temperature increase may affect quite strongly the effective hydrodynamic volume.

To be able to express these ideas on a more quantitative basis more measurements, preferably on more monodisperse PEO samples, are required.

\section{ACKNOWLEDGEMENT}

Thanks are due to G. van de Ridder (Dept. of Chemical Technology) for his careful analysis of the PEO samples.

\section{REFERENCES}

Eshuis, A. and Mellema, J. J. Colloid Polym. Sci. 1984, 262, 159 Eshuis, A., to be published

3 Bailey, F. E. and Koleske, J. V. 'Poly(ethylene oxide)', Academic Press, New York, 1976

4 Sandhu, J. S. and Jit, P. J. Ind. Chem. Soc. 1979, 61, 538

5 Amu, T. C. Polymer 1982, 23, 1775

6 Zhi-Duan H, Xian-Chi Zlang and Rong-Shi Cheng, J. Liq. Chromatography 1982, 5, 1209

7 Oosterbroek, M., Waterman, H. A., Wiseall, S. S., Altena, E. G., Mellema, J. and Kip. G. A. M. Rheologica Acta 1980, 19, 497 Rouse, P. E. J. Chem. Phys. 1953, 21, 1272

Zimm, B. H. J. Chem. Phys. 1956, 24, 269

10 Ferry, J. D. 'Viscoelastic Properties of Polymers', Wiley, New York, 1981, Ch. 9

11 Johnson, R. M., Schrag, J. L. and Ferry, J. D. Polym. J. 1970, 1, 742

12 Elias, H. G. 'Macromolecules', Plenum Press, New York, 1977. pp. $357-358$

13 Mijnlieff, P. F. and Jaspers, W. J. M. Trans. Faraday Soc. 1971 , 67, 582 